\title{
Politique
}

\section{Penser l'État : Gérard Bergeron, un Aufklarer québécois}

\section{Guy Laforest}

Numéro 9, hiver 1986

Démocratie et libéralisme

URI : https://id.erudit.org/iderudit/040518ar

DOI : https://doi.org/10.7202/040518ar

Aller au sommaire du numéro

Éditeur(s)

Société québécoise de science politique

ISSN

0711-608X (imprimé)

1918-6584 (numérique)

Découvrir la revue

Citer cet article

Laforest, G. (1986). Penser l'État : Gérard Bergeron, un Aufklarer québécois. Politique, (9), 147-166. https://doi.org/10.7202/040518ar d'utilisation que vous pouvez consulter en ligne.

https://apropos.erudit.org/fr/usagers/politique-dutilisation/ 


\title{
Penser l'État: Gérard Bergeron, un Aufklarer québécois
}

\author{
Guy Laforest \\ Université McGill
}

Sur le plan théorique ou encore sur celui des essais historiques et conjoncturels, Gérard Bergeron a consacré l'essentiel de sa production intellectuelle à l'étude du politique, et singulièrement à celle de l'étatique. Connue, la place privilégiée qu'occupe cet auteur dans le champ de la science politique québécoise a été rappelée en 1984 par la publication d'un ouvrage de synthèse, Pratique de l'État au Québec, où il a systématiquement réuni pour la première fois les plumes du théoricien et de l'essayiste. À partir d'une lecture critique de cet ouvrage, on se propose dans cet article d'illustrer la pertinence des démarches théoriques et analytiques de Bergeron pour la compréhension du phénomène étatique dans le Québec contemporain. La réflexion procédera en quatre temps: mise en contexte de Bergeron dans le Québec contemporain, présentation des outils théoriques qu'il utilise, illustration de la pertinence de ces outils pour l'étude de l'État québécois, analyse critique de ses postulats en faveur d'une forme de rationalisation et d'un modèle de citoyenneté. Cette dernière section révélera certains points de vue personnels de l'auteur de cet article. En me livrant à l'interprétation des œuvres de Bergeron, je n'échappe 
pas à une réalité incontournable de l'histoire des idées: tout interprète est situé, enraciné, son travail répond dans une large mesure aux exigences de son temps.

\section{Théoriser sur l'État à partir d'ici}

Les dernières phrases de Pratique de l'État au Québec exhalent tout sauf la sérénité tranquille. On y sent encore la morosité postréférendaire:

"Même à l'intérieur des limites constitutionnelles et budgétaires, le Québec reste un État de taille optima pour voir venir, corriger et consolider les acquis. Quant à la vraie "relance», tout le monde sait qu'elle viendra d'ailleurs et qu'elle prendra davantage de temps. Le temps de la patience n'est pas le temps du mépris.

Le mot de la fin qui n'en est pas un «de consolation»: l'État du Québec, qui s'est fait en vingt-cinq ans, reste montrable à l'extérieur et, malgré tout, estimable aux Québécois. L'en-deça et l'au-delà ressortiraient à la critique idéologique et à la philosophie de l'histoire dont ce n'était pas ici le propos" (Bergeron, 1984, p. 429).

«Le Québec reste un État de taille optima»; tout lecteur de Bergeron aura vite compris que le Québec, pour lui, ce n'est pas d'abord un État (Bergeron, 1984, p. 384). C'est principalement une société, faite de citoyens, dotée d'un État comme cela se fait partout dans le monde. Cette proposition nous aide à saisir le contexte fondamental de l'entreprise de Bergeron. La première partie du livre offre un tableau d'ensemble de l'histoire politique du Québec, de plus en plus détaillé à mesure que l'on se rapproche de la période contemporaine. Les deux autres parties analysent respectivement les principales crises traversées par l'État québécois depuis l'avènement du gouvernement péquiste en 1976, et les opérations de fonctionnement de cet État. Je me servirai surtout des deuxième et troisième parties pour illustrer la pertinence théorique de Bergeron, et exclusivement de la troisième pour l'examen critique de ses postulats en faveur de la rationalisation 
et d'un modèle de citoyenneté. Le fait indépassable découlant de l'esquisse introductive, c'est la fragilité de la situation du Québec, société minoritaire culturellement, en un continent où poussent de redoutables forces d'assimilation culturelle et linguistique (Bergeron, 1984, pp. 18 et 46). Le Québec n'aura pas de cesse, dans ces circonstances, avant d'avoir obtenu des garanties générales assurant sa protection et sa spécificité (Bergeron, 1984, p. 118). Pour la génération de Bergeron, la protection, l'affirmation et l'épanouissement du peuple québécois ont passé par le biais de la croissance et de la modernisation de l'État.

Les Québécois ont contrôlé une grande entreprise dans leur histoire: l'État (Bergeron, 1984, p. 144). Pas surprenant que l'impératif de la construction de cet État ait été le principal slogan de la Révolution tranquille. Et comme la reconnaissance de l'autre est tout aussi importante pour les peuples et les communautés qu'elle peut l'être pour les individus, l'activité internationale du Québec ne sera pas la moindre des dimensions de la modernisation étatique; il ne s'agissait de rien de moins que d'un instrument majeur de compensation pour les insécurités d'un destin culturel nord-américain (Bergeron, 1984, pp. 219 et 222). Partageant avec la collectivité dont il était issu, à un moment précis de son histoire, la passion de l'État, Bergeron aura eu le mérite d'assumer son «être au monde», sa liberté située, en s'astreignant à la tâche de la saisie de cet État dans ses multiples facettes.

\section{L'édifice théorique bergeronien}

Le développement de la science politique, d'après Bergeron, exige un travail de conceptualisation théorique. Sommes-nous en présence d'un héritier de l'épistémologie naturaliste et du positivisme logique, influencé par le courant behavioraliste américain en science politique, partisan de l'explication déductive-nomologique et du 
nécessaire pendant prédictif de toute théorie? Trouve-t-on dans la théorie proposée les croyances positivistes habituelles en l'unité de la méthode scientifique et la neutralité axiologique? Pour ces deux questions, le dernier ouvrage de Bergeron accueille favorablement les préceptes de Rickert et Weber, selon lesquels l'activité du savant dans les sciences historico-culturelles s'appuie sur un nécessaire rapport aux valeurs; le savant sélectionne, dans la diversité infinie de la réalité, les faits qui ont une signification culturelle pour lui. Bergeron écrit que la théorie dirige toujours l'entreprise de détermination de l'objet, que «c'est elle qui, devant les flots incessants des faits et événements, sélectionne l'important et même l'essentiel, repère le signifiant et s'attarde au significatif» (Bergeron, 1965, p. 21).

Notre auteur ne peut pas non plus être simplement rangé dans le camp de l'épistémologie naturaliste et du behavioralisme. Même en l'époque de l'optimisme scientiste le plus débridé dans les sciences sociales nord-américaines, il a généralement gardé ses distances devant les virages amorcés par ces disciples vers une mathématisation et une quantification à outrance (Bergeron, 1965, pp. 459-462). Il eût été plutôt satisfait par une science politique "assez mais pas trop "scientifique»" (Bergeron, 1977, p. 10). D'autre part, il n'est pas question pour lui d'expliquer le politique ou l'État, de les enserrer dans un dispositif causal strict, pour proposer ensuite des enchaînements prédictifs garantissant, d'une manière certaine ou probable, des comportements étatique précis de fonctionnement et de transformation. Sous l'appellation d'une théorie de l'État, Bergeron propose des schémas interprétatifs, un type idéal, une visualisation servant à mieux comprendre la diversité étatique et politique dans ses dynamiques de fonctionnement et de changement. La théorie en question ne prétend pas pouvoir se représenter adéquatement ce que l'objet étatique est en lui-même, une fois pour toutes. Plutôt que l'obtention d'un portrait exact, le type suggéré n'affirme rien d'autre que l'utilité 
et la fécondité, théoriques et pratiques, de se représenter l'État de cette manière.

Cette visualisation appartient à une approche que l'on pourrait qualifier de néo-fonctionnalisme physiologique et holiste. C'est un fonctionnalisme parce que l'on met moins l'emphase sur la stabilité institutionnelle que sur la dynamique fonctionnelle la fonction étant une série d'activités liées les unes aux autres en processus d'action, et unifiées par leur commune participation à la vie d'un organisme (Bergeron, 1965, p. 90). C'est aussi un néo-fonctionnalisme parce que l'on accepte explicitement les critiques adressées au fonctionnalisme anthropologique de Malinowski et Radcliffe-Brown, penchant dans le sens de l'indispensabilité des fonctions et de leur nécessaire contribution au maintien d'une société (Bergeron, 1965, pp. 91-92). La démarche de Bergeron mérite cette appellation pour deux autres raisons: elle se distingue d'une insistance sur les conséquences fonctionnelles, à la Merton, pour privilégier la complexité et la richesse des processus fonctionnels eux-mêmes; d'autre part, elle s'inscrira en faux contre la théorie de la séparation ternaire des pouvoirs pour favoriser la division quaternaire des fonctions, au sens restreint, qui sont «les opérations politiques immédiatement décisives»: gouvernement, législation, administration et juridiction (Bergeron, 1965, pp. 144-145). C'est enfin un néo-fonctionnalisme physiologique et holiste, parce qu'il retourne aux sources biologiques du fonctionnalisme, suggérant de visualiser l'État comme une totalité organique dynamique.

En considérant l'État de cette manière, Bergeron ne risquet-il pas de se perdre dans une mer de faits, d'événements et d'institutions, allant des relations internationales aux constitutions des États, des comportements variés des citoyens à la diversité fonctionnelle ci-haut mentionnée? La réponse fournie à cette interrogation est déterminante pour toute la méthode échafaudée par l'auteur. Grâce au concept de niveau, il proclame la valeur 
«d'une technique optique à agencement multiple» pour l'appréhension de cette unité globale de fonctionnement qu'est l'organisme étatique (Bergeron, 1984, p. 423, et Bergeron, 1965, p. 105). Ce faisant, il entre de plein pied dans les façons de la méthodologie wébérienne:

Le niveau est une mise en perspective à détermination objective mais supportant le visionnement toujours en partie subjectif de l'observateur, il laisse donc une certaine part au discrétionnaire de l'analyse. Les éléments retenus sont localisés à tel niveau pour leur qualité d'apparentement et d'homogénéité interactionnelle (Bergeron, 1977, p. 66).

Le tableau ainsi conçu permet d'imaginer une hiérarchisation de la diversité étatique en trois niveaux, sur lesquels l'observateur peut tour à tour s'arrêter à des fins d'analyse, sans jamais perdre de vue l'impossibilité de l'existence d'un niveau à l'extérieur de la totalité organique étatique. Le niveau central, celui de l'activité politique la plus dense, est qualifié de fonctionnel. C'est le domaine de la gouverne politique, dans ses dimensions de commandement, ou "d'impération", avec les fonctions gouvernementales et législative, d'exécution avec les fonctions administrative et juridictionnelle. Venu au fonctionnalisme et à la théorie de l'État par le biais de soucis internationalistes, féru de droit public, Bergeron a intégré dans sa vision les objets traditionnels des études juridiques: la législation et son activité structurante au cœur de sa supériorité juridique, le gouvernement et les mécanismes de ses initiatives décisionnelles lui assurant une primauté politique, l'administration et sa pesanteur organisationnelle, la juridiction et son activité correctionnelle (Bergeron, 1982, pp. 230-235). En se basant sur une analyse serrée de la sémantique et de la sociologie traditionnelle autour des notions de rôle et de contrôle, il a examiné sous tous leurs angles les rapports dynamiques, réversibles et bidirectionnels, entre tous les couples fonctionnels possibles (au sujet des concepts de contrôle et d'interfonctionnalité, voir Bergeron, 1965, pp. 52-72 et 296-297). Le même processus 
relationnel de contrôle s'applique aux activités internes de chacune des fonctions de la gouverne et, on le verra, aux rapports entre les divers niveaux de l'organisme étatique.

Bergeron nous convie à rassembler, au-dessous des mécanismes serrés de la gouverne, la multiplicité du niveau infrafonctionnel, celui de la politie, de la société politique d'où émerge l'État (Bergeron, 1984, pp. 13 et 384). Privilégié en logique de fonctionnement, le niveau de la gouverne cède le pas, en matière de primauté ultime, à celui de la politie. Pas de gouverne, après tout, sans la masse de citoyens capables de lui donner vie, de s'engager avec elle dans une série de comportements allant de la contribution tacite à la rébellion violente, dont Bergeron n'a pas manqué d'esquisser la gradation nuancée (Bergeron, 1977, pp. 3344). Si les citoyens peuvent jouer la carte de la passivité devant l'État, ce dernier ne laisse aucun individu ou groupe à l'écart de ses processus. C'est le principe de l'inclusion obligée, qui saisit selon nous un aspect fondamental de la rationalité politique moderne (Bergeron, 1977, pp. 2-30). Appartiennent aussi à la politie les participations des individus à des groupes d'intérêt, partis politiques et organismes consultatifs.

Les bousculades qui se produisent en politie visent parfois à susciter des transformations au sein du troisième niveau de l'organisme étatique: le régime, ou niveau superfonctionnel. C'est ici que l'organisme étatique est institué en «unité d'organisation politique spécifique», tout en étant distingué de ses pairs via l'entremise des superfonctions de légitimation et de "sécuration" (Bergeron, 1982, p. 229). Avec la légitimation on est sur le terrain de la superfonctionnalité intérieure, puisqu'il s'agit des processus intégrateurs permettant à une unité politique d'agir auprès de ses membres pour justifier tout son être politique et s'assurer la pérennité dans son milieu social; la «sécuration», elle, nous fait entrer dans le domaine de la superfonctionnalité extérieure, 
regroupant les processus auto-protecteurs par l'intermédiaire desquels une unité politique «vise à affirmer et à assurer son existence individuée dans son environnement politique» (Bergeron, 1982, p. 235). Toute la symbolique entourant le chef de l'État, par exemple, s'avère significative pour les deux plans de la superfonctionnalité.

Qui dit pluralité de niveaux entend aussi possibilité de dénivellation. Ce n'est pas la moindre des qualités du type proposé que de permettre une focalisation de l'attention sur ces lieux particulièrement importants que sont d'abord le seuil entre les niveaux du régime et de la gouverne, ensuite et surtout celui entre la politie et la gouverne. Dans le premier cas il s'agit du seuil des «habilitations» mutuelles entre régime et gouverne, dans le second du seuil des "activisations» réciproques entre gouverne et politie (Bergeron, 1977, pp. 107-113 et 117-130). On insistera davantage, plus loin, sur le seuil des "activations". À première vue, la perspective de Bergeron ne semble pas piper les dés en faveur de l'équilibre de l'organisme étatique. Elle insiste évidemment sur les propriétés homéostatiques, d'équilibration et de stabilisation, de la gouverne ou du niveau fonctionnel (Bergeron, 1965, p. 488). Mais sur le plan de la totalité organique étatique l'équilibre est dialectisé, il fait une large place aux contradictions qui se manifestent autant à l'intérieur des niveaux que dans leurs rapports les uns avec les autres.

\section{Pratique de la théorie}

Dans Pratique de l'État au Québec, sa réceptivité à la thématique du changement fournit à l'auteur les outils nécessaires pour la conception d'une typologie des gouvernements qui se sont succédé à Québec depuis 1956. Suit une démonstration de l'utilité de la théorie, et particulièrement de son concept de niveau, dans la 
deuxième partie de l'ouvrage, «L'État du Québec en crises de changement»; pour chaque niveau, l'auteur analyse la principale crise qui l'a traversé depuis l'accession du Parti québécois au pouvoir en 1976. Le lecteur en sort assez familiarisé avec la théorie pour ferrailler avec la troisième partie, qui couvre la moitié du livre, «L'État du Québec en opérations de fonctionnement»; l'essentiel de cette partie est consacré aux aspects superfonctionnels et fonctionnels de l'État du Québec depuis la Révolution tranquille.

Selon Bergeron, trois grandes crises ont rudement secoué la politie québécoise depuis 1976: la crise linguistique, celle portant sur la négociation avec les secteurs public et para-public, enfin l'affrontement référendaire. C'est cette dernière à notre avis qu'il analyse le mieux. Il s'agit pour lui d'une crise au niveau du régime; le débat portait sur la transformation du régime d'État, il fut l'occasion d'une collision frontale entre des principes opposés de légitimation et de sécuration. Grâce à la perspective de l'auteur, on peut concentrer momentanément l'attention sur ce niveau superfonctionnel, accepter d'emblée le caractère conventionnel et partiel de cet isolement, voir comment les autres niveaux interviennent lorsque le régime est placé en figure sur le fond de l’organisme étatique. En poursuivant le raisonnement métaphorique, on peut saisir aussi qu'un centrage des activités dans l'organisme étatique au niveau du régime risque de laisser celui de la gouverne «exsangue», en «latence de fonctionnement». C'est ce qui se passe au Québec lors du référendum.

Dans une écriture dense sont discutés les rôles des processus législatifs et gouvernementaux de même que ceux des partis politiques lors du référendum; on présente les principaux documents autour de l'événement, tels la question, les Livres blanc et beige, le Rapport Pépin-Robarts. Mais surtout Bergeron réussit à monter un récit cohérent parsemé de jugements pondérés. Selon lui, la tenue du référendum fut globalement utile: les Québécois ont 
pu y jauger «le degré de leur propre détermination à se forger un destin collectif de plus grande liberté, ne fût-ce que pour cesser de vivre au-dessus de leurs moyens et de leurs intentions réelles, au plan constitutionnel comme à d'autres» (Bergeron, 1984, p. 196). L'auteur ne manque pas de cynisme, ou de réalisme. Ainsi, il ne croit pas que le gouvernement central eût reconnu un éventuel résultat affirmatif du référendum. Auraient été décisifs la géopolitique nord-américaine et, à l'intérieur du Canada, un refus central de négocier basé sur cette froide «logique arithmétique des nombres nus», presque toujours déterminants en "power politics»(Bergeron, 1984, p. 195).

L'auteur s'aventure aussi dans le labyrinthe que sont les négociations avec les syndicats des secteurs public et para-public. En cette crise de la gouverne, c'est l'occasion pour lui de montrer toute l'amplitude que peuvent avoir les contrôles interfonctionnels entre gouvernement et administration, sans oublier les interventions secondaires mais non négligeables de la législation et de la juridiction. Un élément important de l'originalité de l'organisme étatique québécois y est identifié: les conflits les plus féroces s'y déroulent en plein cœur de l'organisme, en son niveau central, là où l'interfonctionnalité est à son plus dense, entre les deux fonctions à primordialité politique, soit le gouvernement et l'administration. Certes une dimension importante de l'édification de l'État québécois depuis la Révolution tranquille, la modernisation administrative s'est peut-être réalisée avec trop de célébrités; elle a provoqué «une crise de croissance de cet État au métabolisme déficient par hypertrophie administrative mal contrôlée» (Bergeron, 1984, p. 164).

Sur la voie du paroxisme conflictuel se renouvelant tel un cycle infernal tous les trois ans, Bergeron signale les différentes étapes ayant mené à une centralisation presque complète des négociations. L'État québécois, méta-organisation bureaucratisée, 
en est venu à se donner des interlocuteurs tout aussi bureaucratisés et de taille fort enviable, ronronnant comme lui selon les paramètres de la rationalité instrumentale. La résultante fait apparaître le spectre de la létalité pour l'organisme étatique. L'auteur ne manque pas de constater le paradoxe d'une telle intensité conflictuelle vécue, sur le plan individuel, par des citoyens dont les profils sont assez similaires, tels le péquiste et le céquiste moyens (Bergeron, 1984 , p. 163). Avouera-t-on que le lecteur demeure un peu sur sa faim? En se limitant à la visualisation fonctionnelle de l'État dans sa globalité, Bergeron semble avoir choisi de prendre pour acquis les processus de rationalisation particulièrement visibles dans les opérations centrales de la gouverne. Il ne discute pas la place de cette rationalisation dans l'émergence et le développement de l'État moderne, détaillée et critiquée par des courants importants de la pensée socio-politique du vingtième siècle. Il n'est évidemment pas obligé de le faire pour valider la pertinence actuelle de sa perspective. Mais il incite le lecteur à chercher ailleurs des approches faisant preuve d'une plus grande sensibilité historique face à l'émergence des totalités étatiques et des mouvements modernes de rationalisation. C'est l'amorce d'une des principales critiques que nous lui adresserons. Une autre se trouve déjà à l'état embryonnaire dans notre première réaction à l'espèce d'étonnement de Bergeron devant la juxtaposition de la montée aux extrêmes du conflit avec la ressemblance des protagonistes. On chercherait en vain chez lui une reconnaissance du caractère historique, contingent, de ce qui a été appelé la monopolisation du domaine public par des intérêts privés ou des préoccupations sociales limitées à des groupes spécifiques (Arendt, 1961, pp. 31-45).

La troisième grande crise qui secoua le Québec fut linguistique; Bergeron nous livre un bon exposé des raisons culturelles et démolinguistiques de l'intervention de l'État québécois dans ce secteur, du point de vue de la majorité francophone. Il nous fait suivre 
le déroulement des «activations» de la politie à la gouverne menant à une série d'actions législatives et gouvernementales (Bergeron, 1984, pp. 117-142). L'aspect judiciaire de la crise n'y est pas ignoré. On regrettera que l'auteur n'ait pas jugé bon, puisque l'on est selon ses propres mots en crise de la politie, de se pencher sur la trame proprement infrafonctionnelle de l'événement et cela particulièrement du côté de la minorité anglophone depuis la présentation du Livre blanc sur la politique québécoise de la langue française en avril 1977. L'auteur n'ignore pas cette question. Cependant, il laisse passer une bonne occasion de vraiment privilégier le niveau de la politie, sous la forme d'une analyse suivant de près l'émergence d'un groupe d'intérêt, ou moyen d'action préfonctionnalisé, tel Alliance-Québec. Ce qui s'y déroule actuellement, les dialogues qui en émergeront avec la majorité francophone, seront d'une grande importance pour la politie, pour la qualité du débat «autour de ce qu'implique vivre au Québec et être du Québec»(Bergeron, 1984, p. 123).

La perspective de l'auteur fournit le meilleur d'elle-même dans la troisième partie de l'ouvrage, dès sa considération des opérations superfonctionnelles de l'État du Québec. Celles-ci sont à replacer à l'intérieur de la totalité organique canadienne, État fédéral. Bergeron insiste sur le triple étagement du fédéralisme, susceptible de mettre fin au "hold-up» interprétatif qui se dessine dans la foulée du mouvement centralisateur de ces dernières années. Logiquement en fédéralisme, nous dit-il, il faut parler des États fédérés, de l'État central et enfin de l'État fédéral qui les englobe tous (Bergeron, 1984, p. 206). On oublie trop facilement que c'est l'État fédéral en sa collectivité qui octroie à l'État central l'exercice quasi-exclusif de la superfonction de sécuration, tandis que la légitimation est mieux partagée entre États fédérés et central en dépit de la primauté de ce dernier. L'État québécois, au sein de l'entité fédérale, a pu exercer une "sécurisation" de second degré grâce à ses initiatives sur la scène internationale; 
d'autre part son territoire fut l'occasion, en octobre 1970, de la plus grave rébellion en ce siècle le principe de légitimation de l'État fédéral.

C'est dans un chapitre portant sur la constitution et les habilitations dans le niveau du régime que l'on sent toute la force de ce qui fut appelé au début de cet article la morosité postréférendaire. L'auteur y analyse les étapes et le résultat, à ses yeux médiocre, du processus de révision constitutionnelle qui s'est accéléré au Canada depuis une vingtaine d'années. Il le fait assez objectivement, même s'il ne fait pas mystère de ses sympathies envers les efforts québécois pour l'obtention d'un «régime mieux adapté à la politie de cette société culturelle très particulière» (Bergeron, 1984, p. 234). En bonne logique, le Canada de 1985 est une constitution en mal de pays. Pays, le Canada ne peut l'être que d'une manière fédérale. Or, le Québec n'est pas signataire de la Loi constitutionnelle de 1982. Bergeron a raison de citer Duverger: "Il n'y a pas de constitution fédérale sans un accord entre tous les peuples fédérés sur ce pacte social qui fonde la nation ainsi constituée»(Bergeron, 1984, p. 243).

\section{Postulats concernant la rationalisation et la citoyenneté}

L'auteur déploie sa virtuosité analytique dans les chapitres suivants pour examiner tour à tour l'évolution des quatre fonctions de la gouverne au Québec depuis la Révolution tranquille. Ces chapitres témoignent de la grande valeur de sa démarche théorique; le lecteur y entre en disposant d'un prisme lui permettant de comprendre comment «marche» l'État du Québec dans la quotidienneté de son fonctionnement, comment, d'hier à aujourd'hui, les différents axes de la gouverne s'y sont-ils contrôlés les uns les autres. On n'exagèrent pas trop, selon nous, en affirmant que Bergeron voit dans la croissance de l'État et la poussée administrative 
amorcées par la Révolution tranquille un effort quasi-titanesque du Québec pour rattraper la modernité occidentale. Il cite avec approbation le mot d'Eric Weil, selon lequel l'administration est le point d'insertion du raisonnable dans le rationnel de l'État (Bergeron, 1984, p. 294). En l'occurence, de quelle rationalité s'agit-il? Indubitablement, il s'agit de ce que Weber a appelé la "Zweckrationalitat», la rationalité formelle et instrumentale, celle de l'action orientée vers un but, jaugeant les différents moyens pour y parvenir. Pour l'auteur la bureaucratisation de la fonction administrative représente un progrès et une modernisation, la marche de l'administration publique vers une organisation plus rationnelle et plus efficiente.

Bergeron n'est pas un apologiste de la bureaucratie; s'il avalise la supériorité technique de l'exécutant administratif et la nécessité de son intervention, il n'en constate pas moins, sous le rapport de l'interfonctionnalité, la possibilité d'une inversion de l'ordre des primautés entre gouvernement et administration (Bergeron, 1984, pp. 305 et 302). Tout professeur à l'É.N.A.P. qu'il puisse être, il n'a pas renié les valeurs héritées de la génération des fondateurs de la Faculté des sciences sociales de Laval. Il n'est pas rare en ces pages que le citoyen se joigne à l'observateur de l'organisme étatique pour rappeler que cette société se réclame «d'une philosophie politique humaniste»(Bergeron, 1984, p. 340). C'est cela sans doute qui inspire son penchant pour un plus grand contrôle législatif de la réglementation, ses commentaires relatifs à la surabondance des lois-cadres et à ce que cela peut représenter pour l'administration de la justice, son approbation de la démocratisation de l'accès à la justice, des remarques caustiques sur les limites de la prépondérance de la Charte des droits et des libertés de la personne.

Cependant, semblables objectifs et valeurs se marient assez bien chez lui avec les impératifs d'une plus grande efficacité et 
rationalisation administratives. En ce sens, Bergeron fait figure d'Aufklarer québécois, partageant l'optimisme du siècle des Lumières en faveur d'un progrès rationnel sous l'égide de l'Occident. Sur ce point, son mot de la fin, selon lequel la philosophie de l'histoire n'aurait pas ressorti à son propos, tient bien malaisément. Selon notre interprétation, il travaille avec le postulat d'une rationalisation historique croissante; cette affirmation s'appuie sur la place qu'occupe l'État pour lui dans la société, et corrélativement sur le rôle qui est imputé à la rationalisation au sein de l'État. Se glisse à travers cela l'attribution d'un sens à l'histoire, précipitant la réflexion en pleine philosophie de l'histoire.

Globalement, Bergeron semble passer par-dessus les doutes contemporains relatifs à la rationalité et à la modernisation administrative. Mais le lecteur attentif peut souvent deviner une tension, le refus de l'auteur de sombrer dans un optimisme tranquille. On se surprend parfois à retrouver chez lui des formules qui eussent convenu à Foucault, pour lequel l'État moderne avait repris des Églises chrétiennes le flambeau du pouvoir pastoral. Ainsi, Bergeron note les volontés protectrices des super-ministères de l'État péquiste, dont les domaines d'activité s'apparentaient à autant de missions générales (Bergeron, 1984, p. 257, pp. 9899). Il insiste sur la grande dépendance des administrés, sur l'impossibilité de pouvoir attribuer à qui que soit la "gigantisation graduelle mais assez peu ordonnée de l'État» (Bergeron, 1984, pp. 298 et 321). Cette tension n'est jamais plus évidente que dans le chapitre où Bergeron se penche sur les «activations» de la gouverne à la politie.

L'auteur décrit d'abord dans ces pages les processus par lesquels la gouverne parvient à enserrer la politie dans ses filets. La traditionnelle répression y figure en belle position, elle dont on négligerait à tort le raffinement des mécanismes de réhabilitation grâce à une plus grande utilisation des sciences médicales et 
sociales. Mais l'emphase est surtout mise sur les processus de mobilisation et de régulation. On entre dans le premier cas sur le terrain des moyens par lesquels l'État tente de générer la soumission active des citoyens aux normes publiques. S'y bousculent les subtilités du marketing socio-politiques, la volonté de l'État de faire sa propre propagande avec la possibilité de la moduler et de la corriger par l'entremise d'une large utilisation des sondages à consommation interne (Bergeron, 1984, pp. 353-354). De telles techniques font la fierté des sciences sociales modernes. Bergeron ralentit un peu le vent de leur auto-satisfaction en mettant le doigt sur un aspect de la productivité mutuelle entre savoir et pouvoir. Quant à la régulation, elle est opérée par un immense appareil où les agents d'exécution fonctionnelle se chargent de l'application et parfois de la précision des normes préalablement impérées; c'est le processus qui, dans une société, fixe les comportements acceptables. Bergeron décrit cela dans un vocabulaire assez neutre; pour d'autres, l'action des agents juridictionnels et administratifs deviendra un moyen permettant de quadriller la société et d'y traquer les individus. La gouverne active également la politie par le biais des organismes étatiques dits autonomes et via les réseaux para-publics «décentralisés», tels ceux de l'éducation et des affaires sociales. On se contentera de souligner le jugement de l'auteur quant au caractère assez restreint de cette décentralisation; il constate en effet que l'État ne consent à se départir que des pouvoirs d'application et d'exécution, qu'il demeure assez froid face à tout ce qui touche l'amoindrissement de son contrôle, enfin que l'évolution générale au Québec ne semble pas favoriser les autonomies locales.

Fin observateur de l'État québécois, Bergeron modère dans le détail de ses analyses son préjugé fondamental en faveur de la modernisation administrative depuis la Révolution tranquille. Sur une autre dimension toutefois, il prête davantage le flanc à la critique. Le citoyen, la clé en somme de tout l'édifice, doit se 
contenter d'une portion congrue dans la construction de Pratique de l'État au Québec. L'auteur ne lui réserve qu'un chapitre, celui des «activations» de la politie à la gouverne. Y sont surtout considérés les moyens dits préfonctionnalisés, soit les partis politiques, les groupes d'intérêts et les organismes consultatifs. C'est dans ce chapitre que l'auteur révèle un de ses principaux postulats, concernant la nature de la citoyenneté dans les polities modernes:

D'une part parce qu'ils sont beaucoup trop nombreux et, de l'autre, parce que si peu d'entre eux subissent l'attraction de l'exercice direct des rôles divers de la gouverne, les citoyens admettent la nécessité de la substitution, qui est le premier fondement de la légitimité de la représentation. Mais c'est à une double condition de pouvoir nommer au moins les agents d'impération gouvernementale et législative, puisque ceux-ci ont à rendre des comptes et sont susceptibles d'être remplacés. (Bergeron, 1984, pp. 386 et 422 ).

Cette définition de la vie du citoyen telle qu'elle se profile dans les démocraties libérales occidentales est centrale pour toute l'œuvre de Bergeron. Elle est, globalement, correcte. C'est ainsi qu'en Occident les choses en sont venues à se passer. Mais l'auteur ne cède-t-il pas un peu trop vite au danger de l'universalisation du présent occidental, lorsqu'il prétend que sa visualisation s'applique à tout État? À l'intérieur même de la pensée occidentale, la conception d'un citoyen plutôt passif, pour lequel la démocratie se limite à la représentation et à un mécanisme partisan de sélection des gouvernements, est bien loin de jouir d'un quelconque consensus. C.B. Macpherson, par exemple, voit dans le système actuel des partis un complexe oligopolistique capable de définir la demande politique d'une façon semblable à l'action des grandes entreprises capitalistes dans le domaine économique (Macpherson, 1985, p. 115). En écartant cette participation des citoyens nécessaire à leur propre épanouissement moral, le modèle représentatif appauvrirait la vie démocratique. Il n'est pas question ici d'entériner en bloc le modèle de la démocratie de participation. Il suffit tout 
simplement d'indiquer que l'application de ce modèle entraînerait de grandes modifications dans la visualisation de l'État proposée par Bergeron. Elle déplacerait le foyer de la plus grande densité politique de la gouverne à la politie. On peut penser aussi que sa logique inverserait ce qui nous est apparu comme étant la priorité de la gouverne au seuil des activations. La conception de la citoyenneté véhiculée par Bergeron semble donc en faire un théoricien d'un État de la démocratie occidentale d'équilibre, élitiste et pluraliste, où les interactions entre gouverne et politie produisent une adjudication des biens politiques tenant compte de la concurrence entre les individus et les groupes. À ce stadeci on nous objectera, à bon droit, que la perspective de Bergeron inclut également une théorie des changements politiques, qu'elle n'est pas insensible aux arguments que nous venons de développer.

Nous ne pouvons rendre compte ici de cette théorie des changements et de la typologie des gouvernements qui l'accompagne (Bergeron, 1977, pp. 167-183 et Bergeron, 1984, p. 58). Mais en plaçant le premier gouvernement péquiste parmi les régimes de type communaliste, la classe bergeronienne la plus ouverte à la participation et à la citoyenneté, l'auteur nous semble reconnaître implicitement qu'il ne remet pas en question la définition traditionnelle de la citoyenneté en démocratie occidentale. C'est le changement de régime bien plus que l'adoption d'un nouveau modèle de citoyenneté qui fut la principale préoccupation de l'administration Lévesque entre 1976 et 1981.

Nous nous sommes servi, dans cet article, de Pratique de l'État au Québec pour montrer l'utilité de la démarche théorique de Gérard Bergeron. Prise dans les schémas de pensée de l'Occident, la perspective de l'auteur peut servir partout où l'étatique est jouxté aux façons occidentales. En somme, diraient certains, partout. Le système mondial des États est effectivement un produit de ce qu l'on pourrait appeler la mondialisation de l'Occident. C'est 
une autre forme de la dialectique de l'Aufklarung. Bergeron réussit à garder un certain recul face à ce mouvement. Il reconnaît l'enracinement d'une théorie, comme la sienne, dans sa société d'origine. Son néo-fonctionnalisme physiologique et holiste lui fournit également un outil pour entrer dans la complexité de l'État, tout en le protégeant du mirage d'une correspondance parfaite avec la réalité. Aufklarer modéré, Bergeron l'est grâce à des sympathies pour le mouvement occidental de rationalisation, qu'il réussit à freiner dans les jugements critiques parfois portés sur la pratique étatique au Québec.

Nous avons posé des questions aussi au modèle de la citoyenneté proposé par l'auteur. Il semble en effet prendre pour acquis un "homo politicus» qui serait le partenaire indissociable de «l'homo economicus» pensé par les utilitaristes. La complicité entre les deux aurait d'ailleurs pu être démontrée par l'analyse de la politique économique du gouvernement péquiste, énoncée dans un document tel Bâtir le Québec. Il faut bien comprendre que cet aspect n'est pas un défaut du modèle de Bergeron. Ici autant sinon plus qu'ailleurs, sa vision tend à correspondre à ce que les êtres humains sont devenus dans l'histoire. On regrette tout simplement de le voir apporter de l'eau au moulin de la transformation de «l'homo economicus», atomiste, consommateur et hyper-productiviste, et de «l'homo politicus», principalement passif et désintéressé, en structures ontologiques fermes des êtres humains.

\section{Bibliographie}

ARENDT, Hannah, 1961. La condition de l'bomme moderne, Calmann-Lévy, Paris. BERGERON, Gérard, 1984. Pratique de l'État au Québec, Québec/Amérique, Montréal. BERGERON, Gérard, 1982. "L'État et ses trois niveaux: le régime, la gouverne et la politie", Revue canadienne de science politique, XV (2), juin 1982, pp. 227-257. 
BERGERON, Gérard, 1977. La gouverne politique, Mouton et P.U.L., Paris-La Haye et Québec.

BERGERON, Gérard, 1965. Fonctionrement de l'État, Armand Colin et P.U.L., Paris et Québec.

HABERMAS, Jürgen, 1984. The Theory of Communicative Action, vol. I: Reason and the Rationalization of Society, Beacon Press, Boston.

MACPHERSON, C.B., 1985. Principes et limites de la démocratie libérale, Boréal Express/ La Découverte, Montréal. 